PROCEEDINGS OF THE

AMERICAN MATHEMATICAL SOCIETY

Volume 137, Number 5, May 2009, Pages 1631-1639

S 0002-9939(08)09752-9

Article electronically published on November 26, 2008

\title{
THREEFOLDS CONTAINING BORDIGA SURFACES AS AMPLE DIVISORS
}

\author{
HIDETOSHI MAEDA
}

(Communicated by Ted Chinburg)

\begin{abstract}
Let $L$ be an ample line bundle on a smooth complex projective variety $X$ of dimension three such that there exists a smooth member $Z$ of $|L|$. When the restriction $L_{Z}$ of $L$ to $Z$ is very ample and $\left(Z, L_{Z}\right)$ is a Bordiga surface, it is proved that there exists an ample vector bundle $\mathcal{E}$ of rank two on $\mathbb{P}^{2}$ with $c_{1}(\mathcal{E})=4$ and $3 \leq c_{2}(\mathcal{E}) \leq 10$ such that $(X, L)=\left(\mathbb{P}_{\mathbb{P}^{2}}(\mathcal{E}), H(\mathcal{E})\right)$, where $H(\mathcal{E})$ is the tautological line bundle on the projective space bundle $\mathbb{P}_{\mathbb{P}^{2}}(\mathcal{E})$ associated to $\mathcal{E}$.
\end{abstract}

\section{INTRODUCTION}

In this paper varieties are always assumed to be defined over the field $\mathbb{C}$ of complex numbers.

Given a smooth projective variety $Z$, the classification of smooth projective varieties $X$ containing $Z$ as an ample divisor occupies an extremely important position in the theory of polarized varieties, and it is well-known that the structure of $Z$ imposes severe restrictions on that of $X$. Inspired by this philosophy, we set up the following condition $(*)$ :

(*) $L$ is an ample line bundle on a smooth projective variety $X$ such that there exists a smooth member $Z$ of $|L|$.

In this paper we treat Bordiga surfaces $\left(Z, L_{Z}\right)$ under the assumption $(*)$ when the restriction $L_{Z}$ of $L$ to $Z$ is very ample. Here $\left(Z, L_{Z}\right)$ with $L_{Z}$ very ample is called a Bordiga surface if $Z$ is a smooth projective surface obtained by the blowingup $\sigma: Z \rightarrow \mathbb{P}^{2}$ of $\mathbb{P}^{2}$ at $k$ distinct points $p_{1}, \ldots, p_{k}$ in general position $(0 \leq k \leq 10)$ and $L_{Z}=\sigma^{*} \mathcal{O}_{\mathbb{P}^{2}}(4)-\mathcal{O}_{Z}\left(\sum_{i=1}^{k} e_{i}\right)$, where $e_{i}=\sigma^{-1}\left(p_{i}\right)$ for $i=1, \ldots, k$. When $L$ itself is very ample, if $\left(Z, L_{Z}\right)$ is a Bordiga surface, then it follows from II, Theorem 4.2 and Proposition 4.7] and LM2, Lemma 4] that there exists a very ample vector bundle $\mathcal{E}$ of rank two on $\mathbb{P}^{2}$ with $c_{1}(\mathcal{E})=4$ and $3 \leq c_{2}(\mathcal{E}) \leq 10$ such that $(X, L)=\left(\mathbb{P}_{\mathbb{P}^{2}}(\mathcal{E}), H(\mathcal{E})\right)$, where $H(\mathcal{E})$ is the tautological line bundle on the projective space bundle $\mathbb{P}_{\mathbb{P}^{2}}(\mathcal{E})$ associated to $\mathcal{E}$. The purpose of this paper is to generalize the above result when $L$ is simply supposed to be ample. The precise statement of our result is as follows:

Received by the editors November 23, 2007, and, in revised form, July 16, 2008.

2000 Mathematics Subject Classification. Primary 14J25; Secondary 14J30, 14J60.

Key words and phrases. Ample line bundle, Bordiga surface.

(C)2008 American Mathematical Society Reverts to public domain 28 years from publication 
Theorem. Let $L$ be an ample line bundle on a smooth projective variety $X$ of dimension three such that there exists a smooth member $Z$ of $|L|$. Assume that the restriction $L_{Z}$ of $L$ to $Z$ is very ample and that $\left(Z, L_{Z}\right)$ is a Bordiga surface. Then there exists an ample vector bundle $\mathcal{E}$ of rank two on $\mathbb{P}^{2}$ with $c_{1}(\mathcal{E})=4$ and $3 \leq c_{2}(\mathcal{E}) \leq 10$ such that $(X, L)=\left(\mathbb{P}_{\mathbb{P}^{2}}(\mathcal{E}), H(\mathcal{E})\right)$.

This paper is organized as follows. In Section 1 we collect necessary material that will be used later. Sections 2 and 3 are devoted to the proof of the theorem. Concretely, in Section 2, under the assumption in the theorem we prove that there exists an ample vector bundle $\mathcal{E}$ of rank two on $\mathbb{P}^{2}$ with $c_{1}(\mathcal{E})=4$ and $1 \leq c_{2}(\mathcal{E}) \leq 10$ such that $(X, L)=\left(\mathbb{P}_{\mathbb{P}^{2}}(\mathcal{E}), H(\mathcal{E})\right)$. In Section 3 we show that $c_{2}(\mathcal{E}) \geq 3$.

When $\mathcal{E}$ is an ample vector bundle of rank $n-2 \geq 2$ on a smooth projective variety $X$ of dimension $n$ such that there exists a global section $s$ of $\mathcal{E}$ whose zero locus $Z=(s)_{0}$ is a smooth surface on $X$ and $H$ is an ample line bundle on $X$ such that $H_{Z}$ is very ample, the triplets $(X, \mathcal{E}, H)$ are completely classified in [LM1] and LM2 under the assumption that $\left(Z, H_{Z}\right)$ is a Bordiga surface. Consequently the theorem is regarded as a result when $n=3$ and $\mathcal{E}=H$.

Thanks are due to the referee for useful remarks.

\section{Preliminaries}

We use the standard notation from algebraic geometry. The tensor products of line bundles are denoted additively. The pullback $i^{*} \mathcal{E}$ of a vector bundle $\mathcal{E}$ on $X$ by an embedding $i: Y \hookrightarrow X$ is denoted by $\mathcal{E}_{Y}$. In particular, for a closed subvariety $V$ of $\mathbb{P}^{N},\left(\mathcal{O}_{\mathbb{P}^{N}}(1)\right)_{V}$ is denoted by $\mathcal{O}_{V}(1)$. For a vector bundle $\mathcal{E}$ on a projective variety $X$, the tautological line bundle on the projective space bundle $\mathbb{P}_{X}(\mathcal{E})$ associated to $\mathcal{E}$ is denoted by $H(\mathcal{E})$. A vector bundle $\mathcal{E}$ on a projective variety $X$ is said to be ample (respectively very ample) if $H(\mathcal{E})$ is ample (respectively very ample). We denote by $K_{X}$ the canonical bundle of a smooth variety $X$. A polarized manifold is a pair $(X, L)$ consisting of a smooth projective variety $X$ and an ample line bundle $L$ on $X$. The sectional genus $g(X, L)$ of a polarized manifold $(X, L)$ is defined by the formula $2 g(X, L)-2=\left(K_{X}+(n-1) L\right) L^{n-1}$, where $n=\operatorname{dim} X$. A polarized manifold $(X, L)$ is called a scroll over a smooth projective variety $W$ if $(X, L)=\left(\mathbb{P}_{W}(\mathcal{E}), H(\mathcal{E})\right)$ for some ample vector bundle $\mathcal{E}$ on $W$. A polarized manifold $(X, L)$ is called a Del Pezzo manifold if $K_{X}+(\operatorname{dim} X-1) L=\mathcal{O}_{X}$. A pair $(X, L)$ with $L$ very ample is called a Bordiga surface if $X$ is a smooth projective surface obtained by the blowingup $\sigma: X \rightarrow \mathbb{P}^{2}$ of $\mathbb{P}^{2}$ at $k$ distinct points $p_{1}, \ldots, p_{k}$ in general position $(0 \leq k \leq 10)$ and $L=\sigma^{*} \mathcal{O}_{\mathbb{P}^{2}}(4)-\mathcal{O}_{X}\left(\sum_{i=1}^{k} e_{i}\right)$, where $e_{i}=\sigma^{-1}\left(p_{i}\right)$ for $i=1, \ldots, k$.

First let us recall some numerical properties of adjoint bundles.

Lemma 1. Let $L$ be an ample line bundle on a smooth projective variety $X$ of dimension $n \geq 1$.

(i) If $t \geq n+1$, then $K_{X}+t L$ is always nef.

(ii) If $K_{X}+n L$ is not nef, then $(X, L)=\left(\mathbb{P}^{n}, \mathcal{O}_{\mathbb{P}^{n}}(1)\right)$.

(iii) Assume that $K_{X}+n L$ is nef and that $n \geq 2$. If $K_{X}+(n-1) L$ is not nef, then $(X, L)$ is one of the following:

(iii-1) $X$ is a quadric hypersurface $\mathbb{Q}^{n}$ in $\mathbb{P}^{n+1}$, and $L=\mathcal{O}_{\mathbb{Q}^{n}}(1)$;

(iii-2) $(X, L)=\left(\mathbb{P}^{2}, \mathcal{O}_{\mathbb{P}^{2}}(2)\right)$;

(iii-3) $(X, L)$ is a scroll over a smooth projective curve. 
THREEFOLDS CONTAINING BORDIGA SURFACES AS AMPLE DIVISORS

(iv) Assume that $K_{X}+(n-1) L$ is nef and that $n \geq 3$. If $K_{X}+(n-2) L$ is not nef, then $(X, L)$ is one of the following:

(iv-1) there exists an effective divisor $E$ on $X$ such that $\left(E, L_{E},\left(\mathcal{O}_{X}(E)\right)_{E}\right)=$ $\left(\mathbb{P}^{n-1}, \mathcal{O}_{\mathbb{P}^{n-1}}(1), \mathcal{O}_{\mathbb{P}^{n-1}}(-1)\right)$

(iv-2) $(X, L)$ is a Del Pezzo manifold;

(iv-3) $(X, L)=\left(\mathbb{P}^{3}, \mathcal{O}_{\mathbb{P}^{3}}(3)\right)$;

$\left(\right.$ iv-4) $(X, L)=\left(\mathbb{P}^{4}, \mathcal{O}_{\mathbb{P}^{4}}(2)\right)$

(iv-5) $(X, L)=\left(\mathbb{Q}^{3}, \mathcal{O}_{\mathbb{Q}^{3}}(2)\right)$;

(iv-6) $X$ is a $\mathbb{P}^{2}$-bundle over a smooth projective curve $C$, and $L_{F}=\mathcal{O}_{\mathbb{P}^{2}}(2)$ for any fiber $F$ of the bundle projection $X \rightarrow C$;

(iv-7) there exists a surjective morphism $\pi: X \rightarrow C$ onto a smooth projective curve $C$ with Picard number $\rho(C)=\rho(X)-1$ such that any fiber $D$ of $\pi$ is a quadric hypersurface in $\mathbb{P}^{n}$ with $L_{D}=\mathcal{O}_{D}(1)$;

(iv-8) $(X, L)$ is a scroll over a smooth projective surface.

Proof. We refer the reader to [F, Theorems 11.2, 11.7 and 11.8].

Second we need the following:

Lemma 2. Assume that $(X, L)=\left(\mathbb{P}_{C}(\mathcal{E}), H(\mathcal{E})\right)$ for some (not necessarily ample) vector bundle $\mathcal{E}$ of rank $n$ on a smooth projective curve $C$. Then $g(X, L)=g(C)$, where $g(C)$ is the genus of $C$.

Proof. Let $\pi: X \rightarrow C$ be the bundle projection. Then $K_{X}+n L=\pi^{*}\left(K_{C}+\operatorname{det} \mathcal{E}\right)$. Furthermore, the Wu-Chern relation tells us that $L^{n}-L^{n-1} \pi^{*}(\operatorname{det} \mathcal{E})=0$. Thus

$$
\begin{aligned}
& 2 g(X, L)-2=\left(K_{X}+(n-1) L\right) L^{n-1}=\left(-L+\pi^{*}\left(K_{C}+\operatorname{det} \mathcal{E}\right)\right) L^{n-1} \\
& =-L^{n}+L^{n-1} \pi^{*}\left(K_{C}+\operatorname{det} \mathcal{E}\right)=-L^{n-1} \pi^{*}(\operatorname{det} \mathcal{E})+L^{n-1} \pi^{*}\left(K_{C}+\operatorname{det} \mathcal{E}\right) \\
& =L^{n-1} \pi^{*} K_{C}=\operatorname{deg} K_{C}=2 g(C)-2,
\end{aligned}
$$

i.e., $g(X, L)=g(C)$.

Let $(X, L)$ be a Bordiga surface, that is to say, $L$ is a very ample line bundle on a smooth projective surface $X$ obtained by the blowing-up $\sigma: X \rightarrow \mathbb{P}^{2}$ of $\mathbb{P}^{2}$ at $k$ distinct points $p_{1}, \ldots, p_{k}$ in general position $(0 \leq k \leq 10)$, and $L=$ $\sigma^{*} \mathcal{O}_{\mathbb{P}^{2}}(4)-\mathcal{O}_{X}\left(\sum_{i=1}^{k} e_{i}\right)$, where $e_{i}=\sigma^{-1}\left(p_{i}\right)$ for $i=1, \ldots, k$. For $k \geq 1$, the $(-1)$-curves $e_{i}$ satisfy $L e_{i}=1$. Conversely, we also need the following:

Lemma 3. Let $(X, L)$ be a Bordiga surface as above, and let $l$ be a (-1)-curve on $X$ with $L l=1$. Then $l=e_{i}$ for some $i$.

Proof. We refer the reader to [LM1, Proposition 0.2].

In addition, we quote the following from [LM1].

Lemma 4. Let $(X, L)$ be a Bordiga surface as above, let $\rho: X \rightarrow \mathbb{P}^{1}$ be a $\mathbb{P}^{1}$ fibration, and let $f$ be a fiber of $\rho$. Then $f \in\left|\sigma^{*} \mathcal{O}_{\mathbb{P}^{2}}(d)-\mathcal{O}_{X}\left(\sum_{i=1}^{k} m_{i} e_{i}\right)\right|$ for some $d>0$ and for some $m_{i} \geq 0$. Moreover,

$$
\sum_{i=1}^{k} m_{i}^{2}=d^{2} \quad \text { and } \quad \sum_{i=1}^{k} m_{i}=3 d-2 .
$$

Proof. We refer the reader to [LM1, Lemma 0.3].

Finally we prove the following: 
Lemma 5. Let $\mathcal{E}$ be an ample vector bundle of rank $r$ on a smooth projective variety $X$ of dimension $n \geq 2$. Assume that $r \geq n$. If $K_{X}+\operatorname{det} \mathcal{E}$ is not ample, then either $K_{X}+\operatorname{det} \mathcal{E}=\mathcal{O}_{\mathbb{P}^{n}}(-1)$ or $\left(K_{X}+\operatorname{det} \mathcal{E}\right)^{n}=0$.

Proof. If $K_{X}+\operatorname{det} \mathcal{E}$ is not ample, then it follows from [F, Theorems 20.1 and 20.8] that $(X, \mathcal{E})$ is one of the following:

(1) $\left(\mathbb{P}^{n}, \mathcal{O}_{\mathbb{P}^{n}}(1)^{\oplus(n+1)}\right)$;

(2) $\left(\mathbb{P}^{n}, \mathcal{O}_{\mathbb{P}^{n}}(1)^{\oplus n}\right)$;

(3) there exists a vector bundle $\mathcal{F}$ of rank $n$ on a smooth projective curve $C$ such that $X=\mathbb{P}_{C}(\mathcal{F})$, and $\mathcal{E}_{F}=\mathcal{O}_{\mathbb{P}^{n-1}}(1)^{\oplus n}$ for any fiber $F$ of the bundle projection;

(4) $\left(\mathbb{P}^{n}, \mathcal{O}_{\mathbb{P}^{n}}(2) \oplus \mathcal{O}_{\mathbb{P}^{n}}(1)^{\oplus(n-1)}\right)$;

(5) $\left(\mathbb{P}^{n}, T_{\mathbb{P}^{n}}\right)$, where $T_{\mathbb{P}^{n}}$ is the tangent bundle of $\mathbb{P}^{n}$;

(6) $\left(\mathbb{Q}^{n}, \mathcal{O}_{\mathbb{Q}^{n}}(1)^{\oplus n}\right)$.

In cases (1), (4), (5) and (6) we get $K_{X}+\operatorname{det} \mathcal{E}=\mathcal{O}_{X}$. In case (2) we obtain $K_{X}+\operatorname{det} \mathcal{E}=\mathcal{O}_{\mathbb{P}^{n}}(-1)$. Suppose that case (3) holds. Then there exists a vector bundle $\mathcal{G}$ of rank $n$ on $C$ such that $\mathcal{E}=H(\mathcal{F}) \otimes \rho^{*} \mathcal{G}$, where $\rho: X \rightarrow C$ is the bundle projection. We have $K_{X}=-n H(\mathcal{F})+\rho^{*}\left(K_{C}+\operatorname{det} \mathcal{F}\right)$ and $\operatorname{det} \mathcal{E}=n H(\mathcal{F})+$ $\rho^{*}(\operatorname{det} \mathcal{G})$, so that $K_{X}+\operatorname{det} \mathcal{E}=\rho^{*}\left(K_{C}+\operatorname{det} \mathcal{F}+\operatorname{det} \mathcal{G}\right)$. Hence $\left(K_{X}+\operatorname{det} \mathcal{E}\right)^{n}=0$, and the result is proved.

\section{Proof of the theorem: Part I}

Let $\left(Z, L_{Z}\right)$ be a Bordiga surface. Then $Z$ is a smooth projective surface obtained by the blowing-up $\sigma: Z \rightarrow \mathbb{P}^{2}$ of $\mathbb{P}^{2}$ at $k$ distinct points $p_{1}, \ldots, p_{k}$ in general position $(0 \leq k \leq 10)$, and $L_{Z}=\sigma^{*} \mathcal{O}_{\mathbb{P}^{2}}(4)-\mathcal{O}_{Z}\left(\sum_{i=1}^{k} e_{i}\right)$, where $e_{i}=\sigma^{-1}\left(p_{i}\right)$ for $i=1, \ldots, k$. Since $\left(K_{X}+L\right)_{Z}=K_{Z}$ and $K_{Z}$ is not nef, we see that $K_{X}+L$ itself is not nef. Thus it follows from Lemma 1 that $(X, L)$ is one of the following:

(1) $\left(\mathbb{P}^{3}, \mathcal{O}_{\mathbb{P}^{3}}(1)\right)$;

(2) $\left(\mathbb{Q}^{3}, \mathcal{O}_{\mathbb{Q}^{3}}(1)\right)$

(3) $(X, L)$ is a scroll over a smooth projective curve $C$;

(4) there exists an effective divisor $E$ on $X$ such that $\left(E, L_{E},\left(\mathcal{O}_{X}(E)\right)_{E}\right)=$ $\left(\mathbb{P}^{2}, \mathcal{O}_{\mathbb{P}^{2}}(1), \mathcal{O}_{\mathbb{P}^{2}}(-1)\right)$

(5) $(X, L)$ is a Del Pezzo manifold;

(6) $\left(\mathbb{P}^{3}, \mathcal{O}_{\mathbb{P}^{3}}(3)\right)$;

(7) $\left(\mathbb{Q}^{3}, \mathcal{O}_{\mathbb{Q}^{3}}(2)\right)$;

(8) $X$ is a $\mathbb{P}^{2}$-bundle over a smooth projective curve $C$, and $L_{F}=\mathcal{O}_{\mathbb{P}^{2}}(2)$ for any fiber $F$ of the bundle projection $X \rightarrow C$;

(9) there exists a surjective morphism $\pi: X \rightarrow C$ onto a smooth projective curve $C$ with Picard number $\rho(C)=\rho(X)-1$ such that any fiber $D$ of $\pi$ is a quadric surface in $\mathbb{P}^{3}$ with $L_{D}=\mathcal{O}_{D}(1)$;

(10) $(X, L)$ is a scroll over a smooth projective surface $S$.

Furthermore, we have $K_{Z}+L_{Z}=\left(\sigma^{*} \mathcal{O}_{\mathbb{P}^{2}}(-3)+\mathcal{O}_{Z}\left(\sum_{i=1}^{k} e_{i}\right)\right)+\left(\sigma^{*} \mathcal{O}_{\mathbb{P}^{2}}(4)-\right.$ $\left.\mathcal{O}_{Z}\left(\sum_{i=1}^{k} e_{i}\right)\right)=\sigma^{*} \mathcal{O}_{\mathbb{P}^{2}}(1)$, so that $2 g\left(Z, L_{Z}\right)-2=\left(K_{Z}+L_{Z}\right) L_{Z}=$ $\left(\sigma^{*} \mathcal{O}_{\mathbb{P}^{2}}(1)\right)\left(\sigma^{*} \mathcal{O}_{\mathbb{P}^{2}}(4)-\mathcal{O}_{Z}\left(\sum_{i=1}^{k} e_{i}\right)\right)=4$. Hence $g\left(Z, L_{Z}\right)=3$, and we conclude 
that $g(X, L)=3$. Moreover, the Lefschetz theorem tells us that $h^{1}\left(X, \mathcal{O}_{X}\right)=$ $h^{1}\left(Z, \mathcal{O}_{Z}\right)=0$. Set $Z=(s)_{0}$ for some global section $s$ of $L$. Now let us deal with each of the cases (1)-(10) separately.

In case (1) we have $2 g(X, L)-2=\left(K_{X}+2 L\right) L^{2}=\left(\mathcal{O}_{\mathbb{P}^{3}}(-4)+\mathcal{O}_{\mathbb{P}^{3}}(2)\right) \mathcal{O}_{\mathbb{P}^{3}}(1)^{2}=$ -2 , so that $g(X, L)=0$, which contradicts the fact that $g(X, L)=3$.

In case $(2)$ we obtain $2 g(X, L)-2=\left(K_{X}+2 L\right) L^{2}=\left(\mathcal{O}_{\mathbb{Q}^{3}}(-3)+\mathcal{O}_{\mathbb{Q}^{3}}(2)\right) \mathcal{O}_{\mathbb{Q}^{3}}(1)^{2}$ $=-2$, and then $g(X, L)=0$. This is also impossible.

Assume that case $(3)$ holds. Then $h^{1}\left(C, \mathcal{O}_{C}\right)=h^{1}\left(X, \mathcal{O}_{X}\right)=0$, i.e., $C=\mathbb{P}^{1}$. Combining this with Lemma 2, we have $g(X, L)=g(C)=0$. This is absurd because $g(X, L)=3$.

We treat case (4) after case (9). In case (5) we get $2 g(X, L)-2=\left(K_{X}+2 L\right) L^{2}=$ 0 , so that $g(X, L)=1$, which is also impossible.

In case $(6)$ we have $2 g(X, L)-2=\left(K_{X}+2 L\right) L^{2}=\left(\mathcal{O}_{\mathbb{P}^{3}}(-4)+\mathcal{O}_{\mathbb{P}^{3}}(6)\right) \mathcal{O}_{\mathbb{P}^{3}}(3)^{2}=$ 18 , and hence $g(X, L)=10$. This is absurd.

In case $(7)$ we obtain $2 g(X, L)-2=\left(K_{X}+2 L\right) L^{2}=\left(\mathcal{O}_{\mathbb{Q}^{3}}(-3)+\mathcal{O}_{\mathbb{Q}^{3}}(4)\right) \mathcal{O}_{\mathbb{Q}^{3}}(2)^{2}$ $=8$, and so $g(X, L)=5$. This is also absurd.

Now we consider case (8). Then $h^{1}\left(C, \mathcal{O}_{C}\right)=h^{1}\left(X, \mathcal{O}_{X}\right)=0$. Thus $C=\mathbb{P}^{1}$. This directly indicates that $\operatorname{Pic}(X)=\mathbb{Z} \oplus \mathbb{Z}$, and the Lefschetz theorem tells us that $k \geq 1$. We can write $X=\mathbb{P}_{\mathbb{P}^{1}}(\mathcal{E})$, where $\mathcal{E}=\mathcal{O}_{\mathbb{P}^{1}} \oplus \mathcal{O}_{\mathbb{P}^{1}}\left(a_{1}\right) \oplus \mathcal{O}_{\mathbb{P}^{1}}\left(a_{2}\right)$ with $a_{1}, a_{2} \geq 0$. Let $\rho: X \rightarrow \mathbb{P}^{1}$ be the bundle projection, and let $H$ denote the tautological line bundle $H(\mathcal{E})$ on $X$. Then $H$ is spanned. Since $L_{F}=\mathcal{O}_{\mathbb{P}^{2}}(2)$ for any fiber $F$ of $\rho$, we have $L=2 H+b \rho^{*} \mathcal{O}_{\mathbb{P}^{1}}$ (1) for some $b$. Combining [BS, Lemma 3.2.4] with the ampleness of $L$ gives $b>0$. We have $1=L_{Z} e_{1}=\left(2 H_{Z}+b \rho_{Z}^{*} \mathcal{O}_{\mathbb{P}^{1}}(1)\right) e_{1}=$ $2 H_{Z} e_{1}+b\left(\rho_{Z}^{*} \mathcal{O}_{\mathbb{P}^{1}}(1)\right) e_{1}$. Since $H_{Z}$ and $\rho_{Z}^{*} \mathcal{O}_{\mathbb{P}^{1}}(1)$ are spanned, we obtain $H_{Z} e_{1}=0$ and $b=\left(\rho_{Z}^{*} \mathcal{O}_{\mathbb{P}^{1}}(1)\right) e_{1}=1$. Therefore $L=2 H+\rho^{*} \mathcal{O}_{\mathbb{P}^{1}}(1)$. Let us compute the sectional genus $g(X, L)$. Since $K_{X}=-3 H+\rho^{*} \mathcal{O}_{\mathbb{P}^{1}}\left(c_{1}(\mathcal{E})-2\right)$, we get $4=2 g(X, L)-$ $2=\left(K_{X}+2 L\right) L^{2}=\left(-3 H+\rho^{*} \mathcal{O}_{\mathbb{P}^{1}}\left(c_{1}(\mathcal{E})-2\right)+4 H+\rho^{*} \mathcal{O}_{\mathbb{P}^{1}}(2)\right)\left(2 H+\rho^{*} \mathcal{O}_{\mathbb{P}^{1}}(1)\right)^{2}=$ $\left(H+\rho^{*} \mathcal{O}_{\mathbb{P}^{1}}\left(c_{1}(\mathcal{E})\right)\right)\left(4 H^{2}+4 H \rho^{*} \mathcal{O}_{\mathbb{P}^{1}}(1)\right)=4 H^{3}+4+4 c_{1}(\mathcal{E})=8 c_{1}(\mathcal{E})+4$. Hence $c_{1}(\mathcal{E})=0$, i.e., $a_{1}=a_{2}=0$, so that $\mathcal{E}=\mathcal{O}_{\mathbb{P}^{1}}^{\oplus 3}$. Consequently $X=\mathbb{P}^{2} \times \mathbb{P}^{1}$ and $L=\mathcal{O}(2,1)$. However, we can regard $(X, L)$ as $\left(\mathbb{P}_{\mathbb{P}^{2}}\left(\mathcal{O}_{\mathbb{P}^{2}}(2)^{\oplus 2}\right), H\left(\mathcal{O}_{\mathbb{P}^{2}}(2)^{\oplus 2}\right)\right)$. From this, case (8) is included in case (10).

Suppose that $(X, L)$ is as in case $(9)$. Then $h^{1}\left(C, \mathcal{O}_{C}\right) \leq h^{1}\left(X, \mathcal{O}_{X}\right)=0$, so that $C=\mathbb{P}^{1}$. Hence $\rho(X)=2$. By the Lefschetz theorem, the restriction homomorphism $\operatorname{Pic}(X) \rightarrow \operatorname{Pic}(Z)$ is injective. Moreover, $\operatorname{Pic}(Z)$ is torsion free because $Z$ is rational. Thus $\operatorname{Pic}(X)=\mathbb{Z} \oplus \mathbb{Z}$, and hence $k \geq 1$. Set $N=-\left(K_{X}+L\right)$. Then $N_{F}=-\left(K_{F}+L_{F}\right)=\mathcal{O}_{\mathbb{Q}^{2}}(1)$ for a general fiber $F=\mathbb{Q}^{2}$ of $\pi$, and so $\operatorname{Pic}(X)$ is generated by $N$ and $\pi^{*} \mathcal{O}_{\mathbb{P}^{1}}(1)$. Set $L=a N+b \pi^{*} \mathcal{O}_{\mathbb{P}^{1}}(1)$ for some integers $a, b$. Then, since $L_{D}=\mathcal{O}_{D}(1)$ for any fiber $D$ of $\pi$, we obtain $a=1$, so that $L=N+b \pi^{*} \mathcal{O}_{\mathbb{P}^{1}}(1)$. We know that $Z=(s)_{0}$ for some global section $s$ of $L$. Let $s_{F}$ denote the restriction of $s$ to a general fiber $F$. Then $s_{F} \in \Gamma\left(\mathbb{Q}^{2}, \mathcal{O}_{\mathbb{Q}^{2}}(1)\right)$, so that $Z \cap F=\left(s_{F}\right)_{0} \neq \emptyset$. This implies that the restriction $\pi_{Z}: Z \rightarrow \mathbb{P}^{1}$ of $\pi$ to $Z$ is surjective. Now $Z \cap F$ is a conic in $\mathbb{P}^{2}$ for a general $F$, which indicates that $\pi_{Z}$ is a $\mathbb{P}^{1}$-fibration. Set $f=Z \cap F$ for a general $F$. Then $L_{Z}=N_{Z}+b \pi_{Z}^{*} \mathcal{O}_{\mathbb{P}^{1}}(1)=-K_{Z}+b \pi_{Z}^{*} \mathcal{O}_{\mathbb{P}^{1}}(1)=-K_{Z}+$ $b \mathcal{O}_{Z}(f)$. In addition, by Lemma 4 we know that $f \in\left|\sigma^{*} \mathcal{O}_{\mathbb{P}^{2}}(d)-\mathcal{O}_{Z}\left(\sum_{i=1}^{k} m_{i} e_{i}\right)\right|$ for some $d>0$ and for some $m_{i} \geq 0$. Since $-K_{Z}=\sigma^{*} \mathcal{O}_{\mathbb{P}^{2}}(3)-\mathcal{O}_{Z}\left(\sum_{i=1}^{k} e_{i}\right)$, we obtain $L_{Z}=-K_{Z}+b \mathcal{O}_{Z}(f)=\left(\sigma^{*} \mathcal{O}_{\mathbb{P}^{2}}(3)-\mathcal{O}_{Z}\left(\sum_{i=1}^{k} e_{i}\right)\right)+b\left(\sigma^{*} \mathcal{O}_{\mathbb{P}^{2}}(d)-\right.$ $\left.\mathcal{O}_{Z}\left(\sum_{i=1}^{k} m_{i} e_{i}\right)\right)=\sigma^{*} \mathcal{O}_{\mathbb{P}^{2}}(3+b d)-\mathcal{O}_{Z}\left(\sum_{i=1}^{k}\left(1+b m_{i}\right) e_{i}\right)$. On the other hand, 
$L_{Z}=\sigma^{*} \mathcal{O}_{\mathbb{P}^{2}}(4)-\mathcal{O}_{Z}\left(\sum_{i=1}^{k} e_{i}\right)$. Consequently $3+b d=4$ and $1+b m_{i}=1$ for any $i$, so that $b d=1$ and $b m_{i}=0$ for every $i$. Therefore $b=d=1$ and $m_{i}=0$ for any $i$. But then, since $d=1$, it follows from (1.1) that $m_{i}=1$ for some $i$. This is a contradiction.

Let us consider case (4). Let $f: X \rightarrow X^{\prime}$ be the blowing-down of $E$ to a point $p \in X^{\prime}$. Then there exists a line bundle $L^{\prime}$ on $X^{\prime}$ such that $L=f^{*} L^{\prime}-\mathcal{O}_{X}(E)$. It follows from [F, Lemma 7.16] that $L^{\prime}$ is ample on $X^{\prime}$. Set $l=\left(s_{E}\right)_{0}=Z \cap E$. Since $s_{E} \in \Gamma\left(\mathbb{P}^{2}, \mathcal{O}_{\mathbb{P}^{2}}(1)\right), l$ is a linear subspace of $E$ with $\operatorname{dim} l \geq 1$. If $Z \cap E=Z$, then $Z=\mathbb{P}^{2}$, so that $L_{Z}=\left(L_{E}\right)_{Z}=\mathcal{O}_{\mathbb{P}^{2}}(1)$. Thus $K_{Z}+L_{Z}=\mathcal{O}_{\mathbb{P}^{2}}(-2)$, which contradicts the nefness of $K_{Z}+L_{Z}=\sigma^{*} \mathcal{O}_{\mathbb{P}^{2}}(1)$. Hence $l=Z \cap E \varsubsetneqq Z$, and the irreducibility of $Z$ gives $\operatorname{dim} l \leq 1$. Therefore $\operatorname{dim} l=1$, i.e., $l=\mathbb{P}^{1}$. Moreover, $\left(\mathcal{O}_{Z}(l)\right)_{l}=\left(\left(\mathcal{O}_{X}(E)\right)_{Z}\right)_{l}=\left(\left(\mathcal{O}_{X}(E)\right)_{E}\right)_{l}=\mathcal{O}_{\mathbb{P}^{1}}(-1)$. This directly implies that $l$ is a $(-1)$-curve on $Z$. Set $Z^{\prime}=f(Z)$. Then $Z^{\prime}$ is a smooth projective surface, and $Z^{\prime}$ is also a smooth member of $\left|L^{\prime}\right|$. It should be emphasized that $L_{Z}=f_{Z}^{*} L_{Z^{\prime}}^{\prime}-\mathcal{O}_{Z}(l)$, so that $L_{Z} l=1$. Combining this with Lemma 3 leads us to the conclusion that $l=e_{i}$ for some $i$. Consequently $\left(Z^{\prime}, L_{Z^{\prime}}^{\prime}\right)$ is again a Bordiga surface, and $\left(X^{\prime}, L^{\prime}\right)$ satisfies the same assumption as that in the theorem. We have $K_{X}+2 L=f^{*}\left(K_{X^{\prime}}+2 L^{\prime}\right)$ because $K_{X}=f^{*} K_{X^{\prime}}+2 \mathcal{O}_{X}(E)$. Since we are in case (iv-1) of Lemma $1, K_{X}+2 L$ is nef, so that $K_{X^{\prime}}+2 L^{\prime}$ is also nef. Moreover, since $\left(Z^{\prime}, L_{Z^{\prime}}^{\prime}\right)$ is a Bordiga surface, we see that $K_{X^{\prime}}+L^{\prime}$ is not nef. Therefore $\left(X^{\prime}, L^{\prime}\right)$ is as in cases (4)-(10). However, we know that cases (5)-(9) do not occur when $\left(Z^{\prime}, L_{Z^{\prime}}^{\prime}\right)$ is a Bordiga surface (we should keep in mind that case (8) is included in case (10)). Suppose that $\left(X^{\prime}, L^{\prime}\right)$ is as in case (10). Then there exists a smooth rational curve $C$ on $X^{\prime}$ passing through $p$ such that $L^{\prime} C=1$. Let $\widetilde{C}$ be the strict transform of $C$ by $f$. Then $L \widetilde{C}=\left(f^{*} L^{\prime}-\mathcal{O}_{X}(E)\right) \widetilde{C}=L^{\prime} C-\mathcal{O}_{X}(E) \widetilde{C}=0$, which contradicts the ampleness of $L$. Thus $\left(X^{\prime}, L^{\prime}\right)$ must be as in case (4) again. We apply the same argument as above to $X^{\prime}, L^{\prime}$ and $Z^{\prime}$, and continue in this manner. This procedure must come to an end after a finite number of repetitions, and we obtain $(\widetilde{X}, \widetilde{L})$ satisfying the same assumption as in the theorem such that $K_{\widetilde{X}}+\widetilde{L}$ is nef. For the corresponding smooth projective surface $\widetilde{Z}, K_{\widetilde{Z}}$ is nef. This contradicts the fact that $\widetilde{Z}$ is rational, and case (4) does not occur.

Finally we consider case (10). Let $\rho: X \rightarrow S$ be the scroll projection, and let $F$ be an arbitrary fiber of $\rho$. Then $L_{F}=\mathcal{O}_{\mathbb{P}^{1}}(1)$. This indicates that $Z \cap F$ is either a point or all of $F$. In particular, $\rho_{Z}: Z \rightarrow S$ is surjective, so that $\rho_{Z}$ is generically finite. Hence $\rho_{Z}$ is birational. The Lefschetz theorem tells us that the restriction homomorphism $\operatorname{Pic}(X) \rightarrow \operatorname{Pic}(Z)$ is injective, so that $\rho_{Z}$ is not an isomorphism. Thus there exists a positive dimensional fiber $e$ of $\rho_{Z}$. Since $e=Z \cap F$ for some fiber $F$ of $\rho$ and $Z \cap F$ is all of $F$, we have $e=\mathbb{P}^{1}$. We can write $(X, L)=\left(\mathbb{P}_{S}(\mathcal{E}), H(\mathcal{E})\right)$ for some ample vector bundle $\mathcal{E}$ of rank 2 on $S$, and we obtain $K_{X}+2 L=\rho^{*}\left(K_{S}+\operatorname{det} \mathcal{E}\right)$, so that $K_{Z}+L_{Z}=\rho_{Z}^{*}\left(K_{S}+\operatorname{det} \mathcal{E}\right)$. Therefore $0=\left(\rho_{Z}^{*}\left(K_{S}+\operatorname{det} \mathcal{E}\right)\right) e=K_{Z} e+L_{Z} e=K_{Z} e+L_{F} e=K_{Z} e+1$, i.e., $K_{Z} e=-1$. This directly implies that $e$ is a $(-1)$-curve on $Z$. Moreover, since $L_{Z} e=1$, it follows from Lemma 3 that $e=e_{i}$ for some $i$. From this, we can conclude that $S$ is also a smooth projective surface with the Bordiga polarization, so that $\sigma$ factors through $\rho_{Z}$. Let us recall that $K_{Z}+L_{Z}=\rho_{Z}^{*}\left(K_{S}+\operatorname{det} \mathcal{E}\right)$. Since $K_{Z}+L_{Z}=\sigma^{*} \mathcal{O}_{\mathbb{P}^{2}}(1)$ is nef and big, we see that $K_{S}+\operatorname{det} \mathcal{E}$ is also nef and big. Hence by Lemma $5, K_{S}+\operatorname{det} \mathcal{E}$ is ample. We get $\left(K_{Z}+L_{Z}\right) e_{i}=0$ for any $i$ because $K_{Z}+L_{Z}=\sigma^{*} \mathcal{O}_{\mathbb{P}^{2}}(1)$, so that $0=\left(K_{Z}+L_{Z}\right) e_{i}=\left(\rho_{Z}^{*}\left(K_{S}+\operatorname{det} \mathcal{E}\right)\right) e_{i}$ 
for any $i$. Combining this with the ampleness of $K_{S}+\operatorname{det} \mathcal{E}$ implies that $\rho_{Z}\left(e_{i}\right)$ is a point of $S$ for every $i$. Therefore $S=\mathbb{P}^{2}$ and $\rho_{Z}=\sigma$. Thus $K_{X}+2 L=$ $\rho^{*}\left(\mathcal{O}_{\mathbb{P}^{2}}(-3)+\operatorname{det} \mathcal{E}\right)$, which indicates that $K_{Z}+L_{Z}=\rho_{Z}^{*}\left(\mathcal{O}_{\mathbb{P}^{2}}(-3)+\operatorname{det} \mathcal{E}\right)$. We know that $K_{Z}+L_{Z}=\sigma^{*} \mathcal{O}_{\mathbb{P}^{2}}(1)$. Hence $\mathcal{O}_{\mathbb{P}^{2}}(-3)+\operatorname{det} \mathcal{E}=\mathcal{O}_{\mathbb{P}^{2}}(1)$, i.e., $\operatorname{det} \mathcal{E}=\mathcal{O}_{\mathbb{P}^{2}}(4)$. Thus $c_{1}(\mathcal{E})=4$. By the Wu-Chern relation, we have $L^{2}-L \rho^{*} c_{1}(\mathcal{E})+\rho^{*} c_{2}(\mathcal{E})=0$, and hence $L^{3}=L^{2} \rho^{*} c_{1}(\mathcal{E})-L \rho^{*} c_{2}(\mathcal{E})=c_{1}(\mathcal{E})^{2}-c_{2}(\mathcal{E})=16-c_{2}(\mathcal{E})$. On the other hand, $L^{3}=L_{Z}^{2}=\left(\sigma^{*} \mathcal{O}_{\mathbb{P}^{2}}(4)-\mathcal{O}_{Z}\left(\sum_{i=1}^{k} e_{i}\right)\right)^{2}=16-k$. Consequently $c_{2}(\mathcal{E})=k$. Since $\rho_{Z}=\sigma$ is not an isomorphism, we obtain $k \geq 1$, and we conclude that $1 \leq c_{2}(\mathcal{E}) \leq 10$. In Section 3 we show that $c_{2}(\mathcal{E}) \geq 3$.

\section{Proof of the theorem: Part II}

Let $X, L$ and $Z$ be as in the theorem. Then we know that there exists an ample vector bundle $\mathcal{E}$ of rank two on $\mathbb{P}^{2}$ with $c_{1}(\mathcal{E})=4$ and $1 \leq c_{2}(\mathcal{E}) \leq 10$ such that $(X, L)=\left(\mathbb{P}_{\mathbb{P}^{2}}(\mathcal{E}), H(\mathcal{E})\right)$. Let us consider the vector bundle $\mathcal{E} \otimes \mathcal{O}_{\mathbb{P}^{2}}(-2)$. Then $c_{1}\left(\mathcal{E} \otimes \mathcal{O}_{\mathbb{P}^{2}}(-2)\right)=c_{1}(\mathcal{E})+2 c_{1}\left(\mathcal{O}_{\mathbb{P}^{2}}(-2)\right)=0$, so that $\mathcal{E} \otimes \mathcal{O}_{\mathbb{P}^{2}}(-2)$ is normalized in the sense of [OSS, p. 165].

First assume that $\mathcal{E}$ is not semistable. Then OSS, Chapter II, Lemma 1.2.5] tells us that $H^{0}\left(\mathbb{P}^{2}, \mathcal{E} \otimes \mathcal{O}_{\mathbb{P}^{2}}(-3)\right) \neq 0$. Take a nonzero global section $t \in H^{0}\left(\mathbb{P}^{2}, \mathcal{E} \otimes\right.$ $\left.\mathcal{O}_{\mathbb{P}^{2}}(-3)\right)$. If $(t)_{0}=\emptyset$, then we have an exact sequence $\left(\mathcal{E} \otimes \mathcal{O}_{\mathbb{P}^{2}}(-3)\right)^{\vee} \rightarrow \mathcal{O}_{\mathbb{P}^{2}} \rightarrow 0$, where $\left(\mathcal{E} \otimes \mathcal{O}_{\mathbb{P}^{2}}(-3)\right)^{\vee}$ is the dual of $\mathcal{E} \otimes \mathcal{O}_{\mathbb{P}^{2}}(-3)$. Hence the sequence $0 \rightarrow \mathcal{O}_{\mathbb{P}^{2}} \rightarrow$ $\mathcal{E} \otimes \mathcal{O}_{\mathbb{P}^{2}}(-3) \rightarrow \mathcal{O}_{\mathbb{P}^{2}}(-2) \rightarrow 0$ is exact, so that $0 \rightarrow \mathcal{O}_{\mathbb{P}^{2}}(3) \rightarrow \mathcal{E} \rightarrow \mathcal{O}_{\mathbb{P}^{2}}(1) \rightarrow 0$ is exact. Now $\operatorname{Ext}^{1}\left(\mathcal{O}_{\mathbb{P}^{2}}(1), \mathcal{O}_{\mathbb{P}^{2}}(3)\right)=\operatorname{Ext}^{1}\left(\mathcal{O}_{\mathbb{P}^{2}}, \mathcal{O}_{\mathbb{P}^{2}}(2)\right)=H^{1}\left(\mathbb{P}^{2}, \mathcal{O}_{\mathbb{P}^{2}}(2)\right)=0$. Therefore $\mathcal{E}=\mathcal{O}_{\mathbb{P}^{2}}(3) \oplus \mathcal{O}_{\mathbb{P}^{2}}(1)$, and $c_{2}(\mathcal{E})=3$. On the other hand, when $(t)_{0} \neq \emptyset$, we take a line $l$ in $\mathbb{P}^{2}$ such that $\left(t_{l}\right)_{0}=(t)_{0} \cap l$ is a nonempty finite set. Then we can write $\mathcal{E}_{l}=\mathcal{O}_{\mathbb{P} 1}(a) \oplus \mathcal{O}_{\mathbb{P}^{1}}(4-a)$ for some integer $a$. Taking the ampleness of $\mathcal{E}$ and the symmetry into account, we can assume that $a \geq 4-a \geq 1$, so that $2 \leq a \leq 3$. Now $\left(\mathcal{E} \otimes \mathcal{O}_{\mathbb{P}^{2}}(-3)\right)_{l}=\mathcal{O}_{\mathbb{P}^{1}}(a-3) \oplus \mathcal{O}_{\mathbb{P}^{1}}(1-a)$. If $a=3$, then $\left(t_{l}\right)_{0}=l$, which is contrary to our assumption. If $a=2$, then $\left(t_{l}\right)_{0}$ is also $l$. This is still absurd.

Next assume that $\mathcal{E}$ is not stable but semistable. Then by OSS, Chapter II, Lemma 1.2.5] we get $H^{0}\left(\mathbb{P}^{2}, \mathcal{E} \otimes \mathcal{O}_{\mathbb{P}^{2}}(-3)\right)=0$ and $H^{0}\left(\mathbb{P}^{2}, \mathcal{E} \otimes \mathcal{O}_{\mathbb{P}^{2}}(-2)\right) \neq 0$. Take a nonzero global section $t \in H^{0}\left(\mathbb{P}^{2}, \mathcal{E} \otimes \mathcal{O}_{\mathbb{P}^{2}}(-2)\right)$. If $(t)_{0}=\emptyset$, then we obtain an exact sequence $\left(\mathcal{E} \otimes \mathcal{O}_{\mathbb{P}^{2}}(-2)\right)^{\vee} \rightarrow \mathcal{O}_{\mathbb{P}^{2}} \rightarrow 0$, which induces an exact sequence $0 \rightarrow \mathcal{O}_{\mathbb{P}^{2}} \rightarrow \mathcal{E} \otimes \mathcal{O}_{\mathbb{P}^{2}}(-2) \rightarrow \mathcal{O}_{\mathbb{P}^{2}} \rightarrow 0$. As a consequence, the sequence $0 \rightarrow \mathcal{O}_{\mathbb{P}^{2}}(2) \rightarrow \mathcal{E} \rightarrow \mathcal{O}_{\mathbb{P}^{2}}(2) \rightarrow 0$ is exact. We have $\operatorname{Ext}^{1}\left(\mathcal{O}_{\mathbb{P}^{2}}(2), \mathcal{O}_{\mathbb{P}^{2}}(2)\right)=$ $\operatorname{Ext}^{1}\left(\mathcal{O}_{\mathbb{P}^{2}}, \mathcal{O}_{\mathbb{P}^{2}}\right)=H^{1}\left(\mathbb{P}^{2}, \mathcal{O}_{\mathbb{P}^{2}}\right)=0$. Hence $\mathcal{E}=\mathcal{O}_{\mathbb{P}^{2}}(2)^{\oplus 2}$ and $c_{2}(\mathcal{E})=4$. When $(t)_{0} \neq \emptyset$, the case where $\operatorname{dim}(t)_{0}=1$ is impossible because $H^{0}\left(\mathbb{P}^{2}, \mathcal{E} \otimes \mathcal{O}_{\mathbb{P}^{2}}(-3)\right)=0$. Thus $\operatorname{dim}(t)_{0}=0$. Take an arbitrary line $l$ in $\mathbb{P}^{2}$ such that $(t)_{0} \cap l \neq \emptyset$. With the same notation as above we have $\left(\mathcal{E} \otimes \mathcal{O}_{\mathbb{P}^{2}}(-2)\right)_{l}=\mathcal{O}_{\mathbb{P}^{1}}(a-2) \oplus \mathcal{O}_{\mathbb{P}^{1}}(2-a)$. We should keep in mind that $2 \leq a \leq 3$ by the ampleness of $\mathcal{E}$. Thus $a=3$, and $(t)_{0} \cap l$ is a single point $p$ of $\mathbb{P}^{2}$. Therefore $c_{2}\left(\mathcal{E} \otimes \mathcal{O}_{\mathbb{P}^{2}}(-2)\right)=1$, and the Koszul complex gives rise to an exact sequence $0 \rightarrow \mathcal{O}_{\mathbb{P}^{2}} \rightarrow\left(\mathcal{E} \otimes \mathcal{O}_{\mathbb{P}^{2}}(-2)\right)^{\vee}=\mathcal{E} \otimes \mathcal{O}_{\mathbb{P}^{2}}(-2) \rightarrow \mathcal{I}_{p} \rightarrow 0$, where $\mathcal{I}_{p}$ is the ideal sheaf of $p$. Consequently the sequence $0 \rightarrow \mathcal{O}_{\mathbb{P}^{2}}(2) \rightarrow \mathcal{E} \rightarrow \mathcal{I}_{p} \otimes \mathcal{O}_{\mathbb{P}^{2}}(2) \rightarrow 0$ is exact, and $c_{2}(\mathcal{E})=5$.

Finally we assume that $\mathcal{E}$ is stable. Then it follows from OSS, Chapter II, Lemma 1.2.5] that $H^{0}\left(\mathbb{P}^{2}, \mathcal{E} \otimes \mathcal{O}_{\mathbb{P}^{2}}(-2)\right)=0$. We apply the Riemann-Roch theorem to $\mathcal{E} \otimes \mathcal{O}_{\mathbb{P}^{2}}(-1)$. Now $\operatorname{det}\left(\mathcal{E} \otimes \mathcal{O}_{\mathbb{P}^{2}}(-1)\right)=\mathcal{O}_{\mathbb{P}^{2}}(2)$ and $c_{2}\left(\mathcal{E} \otimes \mathcal{O}_{\mathbb{P}^{2}}(-1)\right)=c_{2}(\mathcal{E})-3$. 
The Riemann-Roch theorem tells us that

$$
\begin{aligned}
\chi\left(\mathbb{P}^{2}, \mathcal{E} \otimes \mathcal{O}_{\mathbb{P}^{2}}(-1)\right)= & \frac{1}{2}\left(\operatorname{det}\left(\mathcal{E} \otimes \mathcal{O}_{\mathbb{P}^{2}}(-1)\right)-K_{\mathbb{P}^{2}}\right) \operatorname{det}\left(\mathcal{E} \otimes \mathcal{O}_{\mathbb{P}^{2}}(-1)\right) \\
& =10-c_{2}(\mathcal{E}) .
\end{aligned}
$$

Suppose that $c_{2}(\mathcal{E}) \leq 9$. Then either $h^{0}\left(\mathbb{P}^{2}, \mathcal{E} \otimes \mathcal{O}_{\mathbb{P}^{2}}(-1)\right)>0$ or $h^{2}\left(\mathbb{P}^{2}, \mathcal{E} \otimes\right.$ $\left.\mathcal{O}_{\mathbb{P}^{2}}(-1)\right)>0$. By Serre duality, the latter indicates that $0<h^{0}\left(\mathbb{P}^{2}, K_{\mathbb{P}^{2}} \otimes\right.$ $\left.\mathcal{E}^{\vee} \otimes \mathcal{O}_{\mathbb{P}^{2}}(1)\right)=h^{0}\left(\mathbb{P}^{2}, \mathcal{E} \otimes \mathcal{O}_{\mathbb{P}^{2}}(-6)\right)$. However, since we know that $H^{0}\left(\mathbb{P}^{2}, \mathcal{E} \otimes\right.$ $\left.\mathcal{O}_{\mathbb{P}^{2}}(-2)\right)=0$, we obtain $h^{0}\left(\mathbb{P}^{2}, \mathcal{E} \otimes \mathcal{O}_{\mathbb{P}^{2}}(-6)\right)=0$. Therefore $h^{0}\left(\mathbb{P}^{2}, \mathcal{E} \otimes \mathcal{O}_{\mathbb{P}^{2}}(-1)\right)>$ 0 . Take a nonzero global section $t \in H^{0}\left(\mathbb{P}^{2}, \mathcal{E} \otimes \mathcal{O}_{\mathbb{P}^{2}}(-1)\right)$. If $(t)_{0}=\emptyset$, then we get an exact sequence $\left(\mathcal{E} \otimes \mathcal{O}_{\mathbb{P}^{2}}(-1)\right)^{\vee} \rightarrow \mathcal{O}_{\mathbb{P}^{2}} \rightarrow 0$. Thus the sequence $0 \rightarrow \mathcal{O}_{\mathbb{P}^{2}} \rightarrow \mathcal{E} \otimes$ $\mathcal{O}_{\mathbb{P}^{2}}(-1) \rightarrow \mathcal{O}_{\mathbb{P}^{2}}(2) \rightarrow 0$ is exact, so that $0 \rightarrow \mathcal{O}_{\mathbb{P}^{2}}(1) \rightarrow \mathcal{E} \rightarrow \mathcal{O}_{\mathbb{P}^{2}}(3) \rightarrow 0$ is exact. We have $\operatorname{Ext}^{1}\left(\mathcal{O}_{\mathbb{P}^{2}}(3), \mathcal{O}_{\mathbb{P}^{2}}(1)\right)=\operatorname{Ext}^{1}\left(\mathcal{O}_{\mathbb{P}^{2}}, \mathcal{O}_{\mathbb{P}^{2}}(-2)\right)=H^{1}\left(\mathbb{P}^{2}, \mathcal{O}_{\mathbb{P}^{2}}(-2)\right)=0$, and so $\mathcal{E}=\mathcal{O}_{\mathbb{P}^{2}}(3) \oplus \mathcal{O}_{\mathbb{P}^{2}}(1)$. However, we have $H^{0}\left(\mathbb{P}^{2}, \mathcal{E} \otimes \mathcal{O}_{\mathbb{P}^{2}}(-2)\right)=H^{0}\left(\mathbb{P}^{2}, \mathcal{O}_{\mathbb{P}^{2}}(1) \oplus\right.$ $\left.\mathcal{O}_{\mathbb{P}^{2}}(-1)\right) \neq 0$. This is a contradiction. Moreover, since $H^{0}\left(\mathbb{P}^{2}, \mathcal{E} \otimes \mathcal{O}_{\mathbb{P}^{2}}(-2)\right)=0$, the case where $\operatorname{dim}(t)_{0}=1$ is also impossible. Thus $\operatorname{dim}(t)_{0}=0$. Set $Y=(t)_{0}$. Then $\operatorname{deg} Y=c_{2}\left(\mathcal{E} \otimes \mathcal{O}_{\mathbb{P}^{2}}(-1)\right)=c_{2}(\mathcal{E})-3$. The Koszul complex induces an exact sequence $0 \rightarrow \mathcal{O}_{\mathbb{P}^{2}}(-2) \rightarrow\left(\mathcal{E} \otimes \mathcal{O}_{\mathbb{P}^{2}}(-1)\right)^{\vee}=\mathcal{E} \otimes \mathcal{O}_{\mathbb{P}^{2}}(-3) \rightarrow \mathcal{I}_{Y} \rightarrow 0$, where $\mathcal{I}_{Y}$ is the ideal sheaf of $Y$. Hence the sequence $0 \rightarrow \mathcal{O}_{\mathbb{P}^{2}}(-1) \rightarrow \mathcal{E} \otimes \mathcal{O}_{\mathbb{P}^{2}}(-2) \rightarrow$ $\mathcal{I}_{Y} \otimes \mathcal{O}_{\mathbb{P}^{2}}(1) \rightarrow 0$ is exact. Since $H^{0}\left(\mathbb{P}^{2}, \mathcal{E} \otimes \mathcal{O}_{\mathbb{P}^{2}}(-2)\right)=0$ by assumption and $H^{1}\left(\mathbb{P}^{2}, \mathcal{O}_{\mathbb{P}^{2}}(-1)\right)=0$, we have $H^{0}\left(\mathbb{P}^{2}, \mathcal{I}_{Y} \otimes \mathcal{O}_{\mathbb{P}^{2}}(1)\right)=0$. This means that $Y$ is not contained in a line. Hence $\operatorname{deg} Y \geq 3$, i.e., $c_{2}(\mathcal{E}) \geq 6$. Consequently, if $\mathcal{E}$ is stable, then we see that $c_{2}(\mathcal{E}) \geq 6$.

Thus we conclude that $c_{2}(\mathcal{E}) \geq 3$ when $\mathcal{E}$ is ample with $c_{1}(\mathcal{E})=4$. To sum up, under the assumption in the theorem, there exists an ample vector bundle $\mathcal{E}$ of rank two on $\mathbb{P}^{2}$ with $c_{1}(\mathcal{E})=4$ and $3 \leq c_{2}(\mathcal{E}) \leq 10$ such that $(X, L)=\left(\mathbb{P}_{\mathbb{P}^{2}}(\mathcal{E}), H(\mathcal{E})\right)$. We have completed the proof of the theorem.

The argument developed in this section enables us to prove the following proposition. Statement (3) was proved in $[\mathrm{M}]$ when $\mathcal{E}$ is very ample.

Proposition. Let $\mathcal{E}$ be an ample vector bundle of rank two on $\mathbb{P}^{2}$ with $c_{1}(\mathcal{E})=4$. Then

(1) $c_{2}(\mathcal{E})=3$ if and only if $\mathcal{E}=\mathcal{O}_{\mathbb{P}^{2}}(3) \oplus \mathcal{O}_{\mathbb{P}^{2}}(1)$;

(2) $c_{2}(\mathcal{E})=4$ if and only if $\mathcal{E}=\mathcal{O}_{\mathbb{P}^{2}}(2)^{\oplus 2}$;

(3) $c_{2}(\mathcal{E})=6$ if and only if $\mathcal{E}$ is the cokernel of a bundle monomorphism $\mathcal{O}_{\mathbb{P}^{2}}(1)^{\oplus 2} \rightarrow T_{\mathbb{P}^{2}}^{\oplus 2}$, where $T_{\mathbb{P}^{2}}$ is the tangent bundle of $\mathbb{P}^{2}$.

Proof. The argument developed in this section implies the following when $\mathcal{E}$ is an ample vector bundle of rank two on $\mathbb{P}^{2}$ with $c_{1}(\mathcal{E})=4$ :

(i) $c_{2}(\mathcal{E})=3$ if and only if $\mathcal{E}$ is not semistable;

(ii) $c_{2}(\mathcal{E})=4$ or 5 if and only if $\mathcal{E}$ is not stable but semistable;

(iii) $c_{2}(\mathcal{E}) \geq 6$ if and only if $\mathcal{E}$ is stable.

(1) The "if" part is obvious. Assume that $c_{2}(\mathcal{E})=3$. Then $\mathcal{E}$ is not semistable, so that $\mathcal{E}=\mathcal{O}_{\mathbb{P}^{2}}(3) \oplus \mathcal{O}_{\mathbb{P}^{2}}(1)$.

(2) The "if" part is also obvious. If $c_{2}(\mathcal{E})=4$, then $\mathcal{E}$ is not stable but semistable. Thus we see that $\mathcal{E}=\mathcal{O}_{\mathbb{P}^{2}}(2)^{\oplus 2}$.

(3) Assume that the sequence $0 \rightarrow \mathcal{O}_{\mathbb{P}^{2}}(1)^{\oplus 2} \rightarrow T_{\mathbb{P}^{2}}^{\oplus 2} \rightarrow \mathcal{E} \rightarrow 0$ is exact. Then $\mathcal{E}$ is ample because $T_{\mathbb{P}^{2}}$ is ample. Moreover, $c_{1}(\mathcal{E})=c_{1}\left(T_{\mathbb{P}^{2}}^{\oplus 2}\right)-c_{1}\left(\mathcal{O}_{\mathbb{P}^{2}}(1)^{\oplus 2}\right)=4$, 
and $c_{2}(\mathcal{E})=c_{2}\left(T_{\mathbb{P}^{2}}^{\oplus 2}\right)-c_{1}(\mathcal{E}) c_{1}\left(\mathcal{O}_{\mathbb{P}^{2}}(1)^{\oplus 2}\right)-c_{2}\left(\mathcal{O}_{\mathbb{P}^{2}}(1)^{\oplus 2}\right)=6$. Hence it suffices to prove the "only if" part.

Suppose that $\mathcal{E}$ is an ample vector bundle of rank two on $\mathbb{P}^{2}$ with $c_{1}(\mathcal{E})=4$ and $c_{2}(\mathcal{E})=6$. Then $\mathcal{E}$ is stable. Let us consider the vector bundle $\mathcal{E} \otimes \mathcal{O}_{\mathbb{P}^{2}}(-2)$, which is also stable. Then $c_{1}\left(\mathcal{E} \otimes \mathcal{O}_{\mathbb{P}^{2}}(-2)\right)=c_{1}(\mathcal{E})+2 c_{1}\left(\mathcal{O}_{\mathbb{P}^{2}}(-2)\right)=0$, and $c_{2}\left(\mathcal{E} \otimes \mathcal{O}_{\mathbb{P}^{2}}(-2)\right)=c_{2}(\mathcal{E})+c_{1}(\mathcal{E}) c_{1}\left(\mathcal{O}_{\mathbb{P}^{2}}(-2)\right)+c_{1}\left(\mathcal{O}_{\mathbb{P}^{2}}(-2)\right)^{2}=2$. It follows from the Beilinson spectral sequence that $\mathcal{E} \otimes \mathcal{O}_{\mathbb{P}^{2}}(-2)$ is the cokernel of a bundle monomorphism $\mathcal{O}_{\mathbb{P}^{2}}(-1)^{\oplus 2} \rightarrow\left(\Omega_{\mathbb{P}^{2}}^{1} \otimes \mathcal{O}_{\mathbb{P}^{2}}(1)\right)^{\oplus 2}$ [OSS, Example 2, p. 248]. Since $\Omega_{\mathbb{P}^{2}}^{1}=T_{\mathbb{P}^{2}} \otimes \mathcal{O}_{\mathbb{P}^{2}}(-3)$, the sequence

$$
0 \rightarrow \mathcal{O}_{\mathbb{P}^{2}}(-1)^{\oplus 2} \rightarrow\left(T_{\mathbb{P}^{2}} \otimes \mathcal{O}_{\mathbb{P}^{2}}(-2)\right)^{\oplus 2} \rightarrow \mathcal{E} \otimes \mathcal{O}_{\mathbb{P}^{2}}(-2) \rightarrow 0
$$

is exact. This directly leads us to the conclusion that $\mathcal{E}$ is the cokernel of a bundle monomorphism $\mathcal{O}_{\mathbb{P}^{2}}(1)^{\oplus 2} \rightarrow T_{\mathbb{P}^{2}}^{\oplus 2}$.

\section{REFERENCES}

[BS] M. C. Beltrametti and A. J. Sommese, The Adjunction Theory of Complex Projective Varieties, de Gruyter Exp. Math., vol. 16, de Gruyter, Berlin, 1995. MR1318687 (96f:14004)

[F] T. Fujita, Classification Theories of Polarized Varieties, London Math. Soc. Lecture Note Ser., vol. 155, Cambridge Univ. Press, Cambridge, 1990. MR.1162108(93e:14009)

[I] P. Ionescu, Embedded projective varieties of small invariants, Algebraic Geometry, Bucharest, 1982 (L. Bădescu and D. Popescu, eds.), Lecture Notes in Math., vol. 1056, Springer, Berlin, 1984, pp. 142-186. MR749942 (85m:14024)

[LM1] A. Lanteri and H. Maeda, Ample vector bundles and Bordiga surfaces, Math. Nachr. 280 (2007), 302-312. MR2292152(2008b:14072)

[LM2] A. Lanteri and H. Maeda, Projective manifolds of sectional genus three as zero loci of sections of ample vector bundles, Math. Proc. Cambridge Philos. Soc. 144 (2008), 109118. MR2388237

[M] H. Maeda, The threefold containing the Bordiga surface of degree ten as a hyperplane section, Math. Proc. Cambridge Philos. Soc. 145 (2008), 619-622.

[OSS] C. Okonek, M. Schneider and H. Spindler, Vector Bundles on Complex Projective Spaces, Progr. Math., vol. 3, Birkhäuser Boston, Boston, MA, 1980. MR561910 (81b:14001)

Department of Mathematics, Faculty of Science and Engineering, Waseda UniverSiTy, 3-4-1 Ohkubo, Shinjuku-Ku, TOKYo 169-8555, Japan

E-mail address: maeda@variety.sci.waseda.ac.jp 